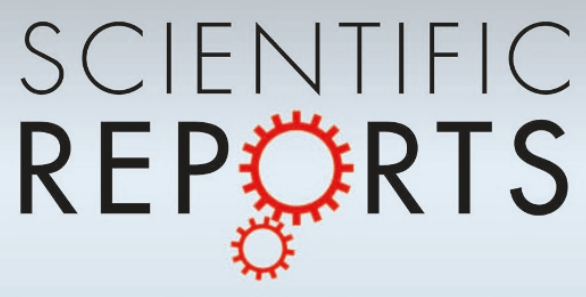

OPEN

SUBJECT AREAS:

GLYCOMICS

EPIDEMIOLOGY

BIOCHEMICAL NETWORKS

PREDICTIVE MARKERS

Received

17 July 2014

Accepted

20 November 2014

Published

23 January 2015

Correspondence and requests for materials should be addressed to Z.-F.Y. (jeffyah@163. com) or Z.-H.J. (zhiiang@must.edu. $\mathrm{mol}$

* These authors contributed equally to this work.

\section{Glycomic Signatures on Serum lgGs for Prediction of Postvaccination Response}

\author{
Jing-Rong Wang ' *, Wen-Da Guan ${ }^{2 *}$, Lee-Fong Yau', Wei-Na Gao' , Yang-Qing Zhan ${ }^{2}$, Liang Liu', \\ Zi-Feng Yang ${ }^{2} \&$ Zhi-Hong Jiang ${ }^{1,2}$
}

\begin{abstract}
'State Key Laboratory of Quality Research in Chinese Medicine, Macau Institute for Applied Research in Medicine and Health, Macau University of Science and Technology, Macau, China, ${ }^{2}$ State Key Laboratory of Respiratory Disease, National Clinical Research Center for Respiratory Disease, First Affiliated Hospital of Guangzhou Medical University, Guangzhou, Guangdong, China.
\end{abstract}

Millions of individuals are vaccinated worldwide each year to stimulate their adaptive immune systems to produce protective antibodies and T-cell response against pathogens. Since glycosylation of the Fc region of immunoglobulin G (IgG) can be influenced by the host's immune status, it was inferred that glycosylation profile of IgG might be altered as a result of the immune response. Therefore, subclass-specific glycosylation profiles of serum IgGs from 26 healthy adults before and after vaccination with a trivalent subunit influenza virus vaccine were comprehensively analyzed to explore glycomic signatures for vaccination. The results showed that no significant changes in the glycosylation of total IgGs took place before and after vaccination, but distinct glycosylation profiles in responders (fourfold or more increase of $\mathrm{HI}$ titer after vaccination) and nonresponders (less than fourfold increase of HI titer) were observed. This difference between the responders and nonresponders occurred even in the resting state. On the basis of variable importance parameters, glycosylation markers that distinguish responders from nonresponders were identified. These markers can be used as molecular signatures to predict antibody titers after vaccination. This is the first study of serum IgG glycosylation profiles in healthy adults receiving a trivalent inactivated influenza vaccine.

nfluenza viruses are respiratory pathogens that cause high morbidity and mortality worldwide every year. Vaccination is one of the most effective methods to prevent influenza infection. The composition of vaccines is recommended annually by the World Health Organization (WHO) to ensure induced immune response against epidemic strain effectively ${ }^{1}$. However, systematic review and meta-analysis on the efficacy and effectiveness of influenza vaccines have shown that pooled efficacy of trivalent inactivated influenza vaccine (TIV) was only 59\% in adults aged 18-65 years, which indicated a moderate protection of influenza vaccines against virologically confirmed influenza ${ }^{2}$. The protective effects of the immune response are influenced not only by the antigenic match between the vaccine strains and epidemic strains ${ }^{3}$, but also by the immunocompetence of individuals ${ }^{4-7}$. Hence, the identification of markers that can accurately predict vaccination efficacy is a pressing need, of which the information would not only enhance the efficacy of current vaccines through personalized vaccination approaches, but would also aid in exploring important mechanisms affecting efficacy, which would in turn contribute to the rational development of next-generation vaccines ${ }^{8}$.

Immunoglobulin $\mathrm{G}(\mathrm{IgG})$ is a major antibody isotype in the blood that can protect the body from infection of pathogens. The Fab region of the IgG molecule is responsible for recognizing and binding to non-self antigens, whereas the Fc region implements elimination of foreign substances by interacting with complement molecules and $\mathrm{Fc}$ receptors to activate the complement system and induce antibody-dependent cell-mediated cytotoxicity $(\mathrm{ADCC})^{9,10}$. The N-glycan located in the Fc region has been shown to affect the binding affinity of IgG to Fc receptors and complement components ${ }^{11-14}$. Glycosylation pattern of Fc determined by the ratio of B cells with different types of glycosyltransferases varies between different individual IgG molecules ${ }^{15}$. When B cells are stimulated by "environmental" factors, mediators that stimulate the innate immune system, or factors arising from the adaptive immune system, Fc glycosylation could be modulated as a result of significant changes in the expression of glycosylation genes ${ }^{16,17}$. Since 1985, a series of studies have shown that the glycosylation of the Fc region can be used to identify a certain number of autoimmune and inflammatory diseases (e.g. rheumatoid arthritis). The glycosylation of IgG has also been shown to correlate with disease progression and clinical outcome $^{12,18-24}$. These data implicit a close association between variations in the glycosylation of IgG and changes in the immune status of humans. 
On the basis of aforementioned, it can be speculated that glycosylation of IgG might be altered as a result of the immune response. It has been reported that glycosylation of antigen-specific IgG1 changed upon monovalent influenza vaccination ${ }^{25}$. A recent study on the temporal response patterns to influenza vaccine revealed a distinct human plasma cell gene signature in subject-specific manner after trivalent influenza vaccination ${ }^{25,26}$. Moreover, early molecular signatures of vaccination for seasonal influenza in humans were recently investigated by using a system biology approach, so as to provide early signatures that correlated with and can be used to predict immune responses after vaccination. Even though, few reports have shown whether the immune response to vaccination correlate with glycosylation patterns of serum IgG, especially at baseline level. In order to understand the normal changes in IgG glycosylation in response to TIV, and to explore glycomic signatures for predication of vaccination efficacy, we herein analyzed the glycosylation of serum IgG and the immune responses of recipients of TIV to compare the levels of specific IgG glycans in responders and nonresponders, and to establish discernible glycosylation patterns for predicting the immune response.

\section{Results}

Variations in antibody titers before and after vaccination. We evaluated antibody responses of 26 healthy adult healthcare workers in our university hospital. The hemagglutination inhibition (HI) titers for each of the three components of the vaccine were determined in the sera from the inoculated subjects before (day 0) and after vaccination (day 14). Some participants had specific antibodies against the three influenza strains before vaccination (the seroprevalence rates of the antibodies to $\mathrm{H} 1, \mathrm{H} 3$ and B were $46.2 \%, 96.2 \%$, and $34.6 \%$, respectively) (Figure 1A). After inoculation, the seroprevalence rates of their antibodies directed against the $\mathrm{H} 1$ and $\mathrm{B}$ vaccine strains increased significantly (by $100 \%$ and $84.6 \%$, respectively) (Figure $1 \mathrm{~A}$ ). A prevaccination $\mathrm{HI}$ titer $<1: 10$ and a post-vaccination $\mathrm{HI}$ titer $>1: 40$ or a pre-vaccination HI titer $>1: 10$ and a minimum fourfold increase 14 days after vaccination are defined as "seroconversion", according to the guidance for the license of pandemic influenza vaccines provided by the US Food and Drug Administration ${ }^{27}$. Accordingly, the vaccinees were classified as "responders" and "nonresponders" on the basis of whether a fourfold increase of the

A

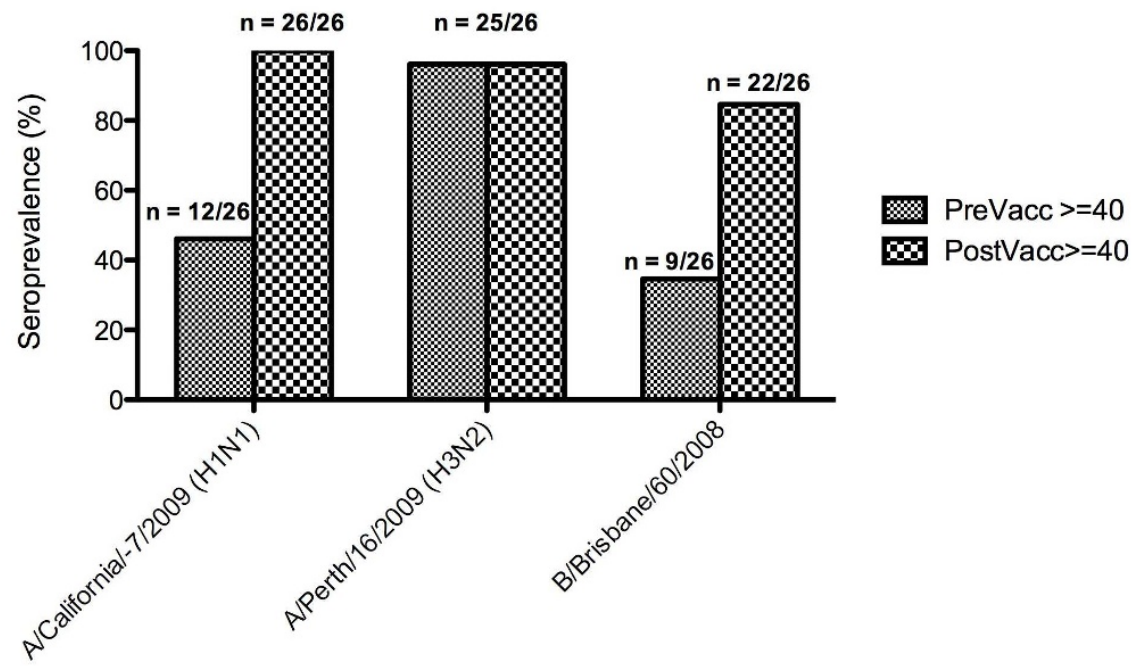

Seasonal influenza vaccine strains

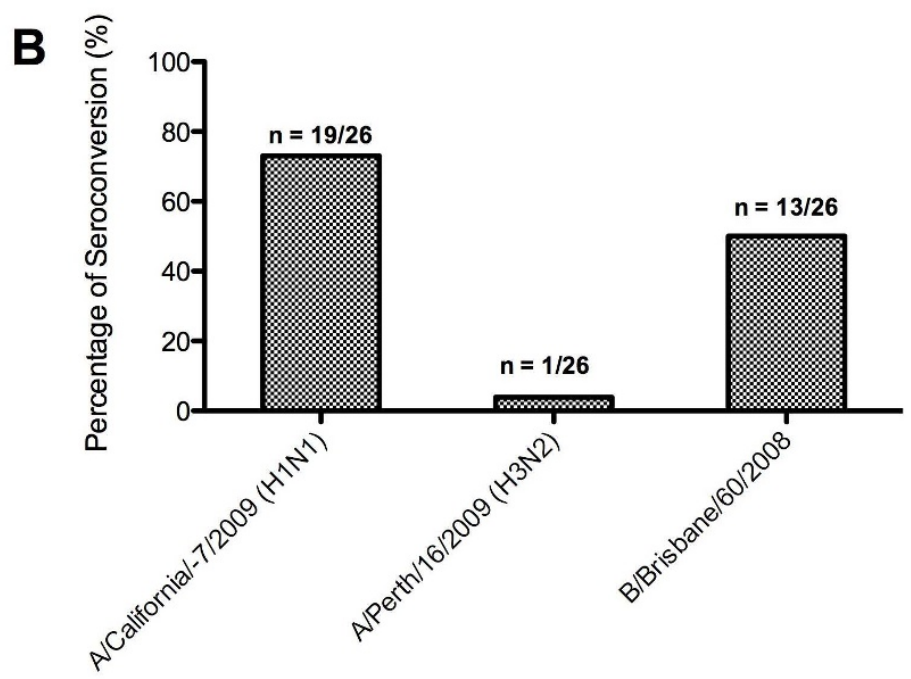

Seasonal influenza vaccine strains

Figure $1 \mid$ (A) Seroprevalence (\%) of HI antibodies (titers $>=40$ ) before and 14 days after vaccination. (B) Sufficient immune response (defined as seroconversion induced by the vaccine) after vaccination. 
HI titer for specific influenza strain was observed after vaccination. As can be seen from Figure 1B, there was considerable variation in the magnitude of the HI responses determined for each of the influenza strains in TIV in the serum of vaccinees. The percentage seroconversion of the $\mathrm{H} 1$ strain was significantly higher $(73.1 \%)$ than that of the H3 strain (3.8\%) or the B strain $(50.0 \%)$ in these subjects.

As observed in other influenza vaccination studies, a correlation between the initial $\mathrm{HI}$ titers (day 0 ) and the antibody response after vaccination was detected in our study (Supporting information, Figure S1A, B). We also analyzed the correlation between the immune response and age or sex. A significant difference in the average age of the responders and nonresponders to the $\mathrm{B}$ strain was observed $(P=0.003$; Table 1$)$.

Questionnaire survey results. All 26 research subjects (13 men, 13 women) returned valid questionnaires, representing a response rate of $100 \%$. The average age of the participants was $33.3 \pm 10.8$ years (range: $24-52$, interquartile range $=26-37.5$, Table 2). Only two subjects had underlying condition or history of influenza-like illness (ILI) symptoms (one with tuberculosis had developed ILI symptoms, and another one sought outpatient service for ILI symptoms twice within one year after vaccination).

Subclass-specific glycosylation analysis of human sera. A comprehensive analysis of the glycosylation of four subclasses of serum IgG in humans after vaccination against influenza was performed using ultra-high performance liquid chromatography coupled with electrospray ionization triple quadrupole mass spectrometer (UHPLC-ESI-QqQ MS)-based multiple reaction monitoring (MRM) method established in our laboratory (to be published elsewhere). Polyclonal IgGs (IgG1, IgG2/3 and IgG4) were isolated from sera with Protein G Sepharose ${ }^{\mathrm{TM}}$ Fast Flow beads in microcentrifuge tubes. Subclass-specific glycosylation analyses at the glycopeptide level were conducted after trypsin digestion, with the resulting glycopeptides analyzed for glycosylation profiling by using UHPLC coupled with electrospray ionization quadrupole time-of-flight mass spectrometer (UHPLCESI-Q-TOF MS). Since the glycopeptides derived from individual IgG subclasses have different molecular weights and distinct retention time on $\mathrm{C}_{18}$ column, this allowed the unambiguous assignment of the glycoforms at the level of subclass-specific IgGs. The characterized glycopeptides were quantified with the MRM technique by using fragment ions generated from the innermost $\mathrm{N}$-acetylglucosamine (GlcNAc) $(\mathrm{m} / z$ 204.1) or hexose-GlcNAc $(\mathrm{m} /$ $\mathrm{z}$ 366.1) residue as product ion, regardless of the sequences of the peptides. In total, 74 glycopeptides derived from the IgG1, IgG2/ IgG3, and IgG4 subtypes were characterized and well quantified using this approach (Figure 2, Table 3). The glycosylation of each individual subject was evaluated using the relative abundance of each glycoform. This method allowed the detailed glycosylation analysis of IgG in human serum before and after vaccination.
Table 2 | Responses to the questionnaire about the efficacy of the influenza vaccine during the first year

\section{Description} Subject Number (\%)

\section{Current smoker}

History of secondhand smoker

Underlying conditions

Cardiac disease

Lung disease ${ }^{\dagger}$

Diabetes mellitus

Cerebral apoplexy

Tuberculosis

Pneumoconiosis

Other chronic diseases

Developed ILI symptoms during the first year after vaccination $\S \S$

Notes: † Lung diseases are defined as chronic obstructive pulmonary disease, asthma, bronchiectasis, interstitial lung disease, and cancer. +Other chronic diseases affecting the renal system, endocrine system (excluded diabetes), hematological system, nervous system (excluding cerebrovascular disease), and gastrointestinal system. §§ ILI symptoms were defined as fever (> $38^{\circ} \mathrm{C}$ ) with two or more of the following symptoms: headache, chill, myalgia or joint pain, fatigue, coryza, sore throat and dry cough.

Glycosylation of IgG before and after vaccination. To observe the changes in IgG glycosylation after vaccination, the quantitation data for the individual glycopeptides derived from $\operatorname{IgG} 1, \operatorname{IgG} 2 / 3$, and IgG4 were first visualized using principal components analysis (PCA) and partial least-squares-discriminant analysis (PLS-DA). Both the PCA and PLS-DA scores plots showed indistinguishable distributions of samples before and after vaccination, suggesting that there was no significant difference between the samples before and after vaccination in either the responder (Figure $3 \mathrm{~B}, \mathrm{E}$ ) or nonresponder group (Figure 3A, D). Detail comparisons showed that relative abundance of most glycoforms of specific subject before and after vaccination were relatively stable across the time span of 14 days (Figure 4).However, when using PLS-DA model, a trend separating the responders from the nonresponders to either FluB (Figure 3C) or H1N1 (Figure 3F) was observed, despite the samples were collected before or after vaccination. This implied that glycosylation was differentially expressed in responders and nonresponders.

Multivariate analysis of glycosylation profiles based on the antibody response. Since a separation trend between responders and nonresponders was observed, a further orthogonal partial least squares discriminate analysis (OPLS-DA) analysis was performed, which revealed an obvious separation between responders and nonresponders, regardless of whether the samples were collected before or after vaccination (Figure 5A, B). For responders and nonresponders to FluB, $Q^{2} Y$ was calculated to be $71 \%$ (four significant components, $\mathrm{R}^{2} \mathrm{Y}=90 \%$; Figure $5 \mathrm{~B}$ ) in the OPLS-DA model, suggesting the good predictive capacity of the model. The major differences in glycosylation between the responders and

Table 1 | Characteristics of participants with or without immune response after vaccination

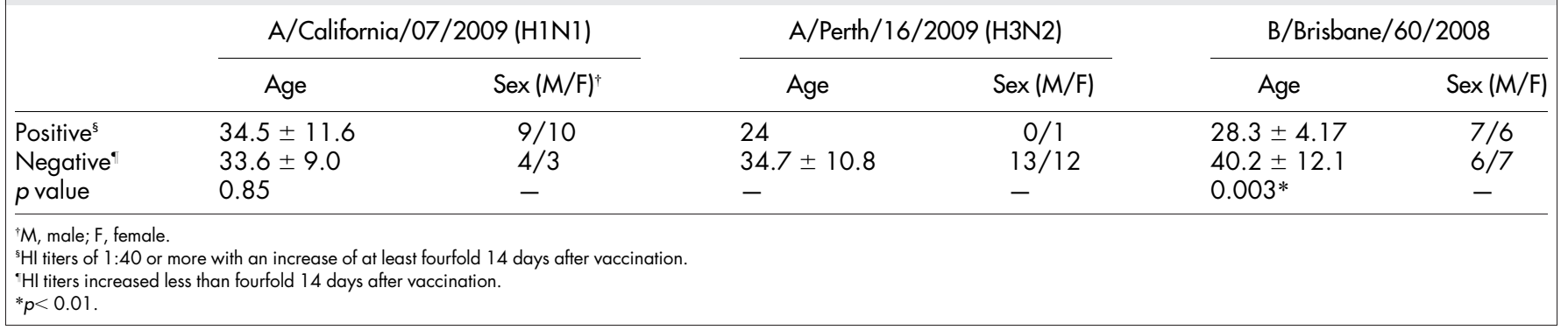


nonresponders were determined from the loading plot and the VIP values for the OPLS-DA model. The potential glycosylation markers were confirmed using a univariate statistical analysis. As could be seen in Figure 6, differentiated glycoforms were predominantly associated with truncated glycans of IgG1. High mannose, sialylated and bisecting glycans were also involved. These results indicated that IgG glycosylation correlated with the immune response and might be used to predict antibody titers for FluB after vaccination.

The correlation between IgG glycosylation and the HI test for $\mathrm{H} 1 \mathrm{~N} 1$ was also examined with the same multivariate analysis as that used for FluB group. As was observed for FluB, the OPLS-DA scores model showed a clear separation between responders and nonresponders (Figure 5C) with a high $\mathrm{Q}^{2}$ value $(68.6 \%$, five significant components, $R^{2} Y=92 \%$; Figure $5 D$ ), suggesting differential glycosylation patterns between the responders and nonresponders. The specific glycoforms contributing to the separation observed in the OPLSDA scores plots were examined with the corresponding loading plot. Potential glycosylation markers with VIP $>1$ are shown in Figure 7.
Because of the observed correlation between baseline titers (day 0 ) and the vaccination response (day 14) in our data, the different glycosylation profiles between responders and nonresponders might be attributed to variation in the initial titers. Thus we analyzed the correlation between baseline antibody titers and the relative levels of each glycoform marker for the prediction of vaccination response. Consequently, only weak correlations between the initial titers and the relative levels of 2 glycoform markers for H1 strain (IgG1-G1FS and IgG1-G1N-a, Supporting information, Figure S3) were observed, but no any correlation was detected for glycomic signatures of FluB (Figure S2). This indicated that the Fc glycosylation profiles may not be simply correlated with baseline antibody titer, which was further supported by PLS-DA and OPLS-DA analyses of global glycosylation profiles based on the initial antibody titers (data not show).

\section{Discussion}

In the past years, several independent groups have used systems biology to predict the immune responses in humans vaccinated against influenza or yellow fever by identifying early gene 'signa-
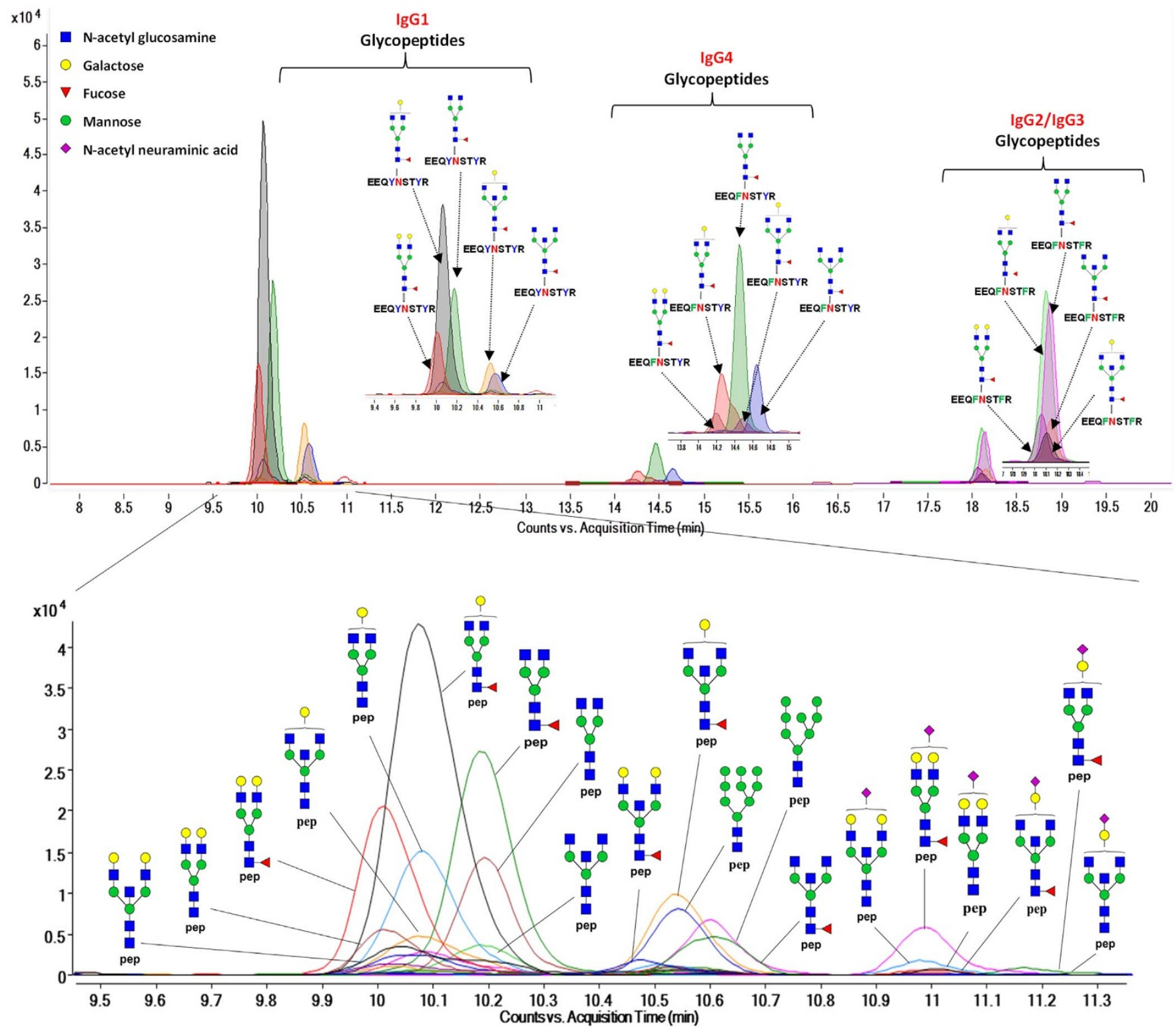

Figure $2 \mid$ MRM chromatogram of representative glycopeptides from different IgG subclasses in human serum. Monosaccharide symbols are based on the Consortium for Functional Glycomics (CFG): blue square, $\mathrm{N}$-acetylglucosamine; yellow circle, galactose; red triangle, fucose; green circle, mannose; purple diamond, $\mathrm{N}$-acetyl neuraminic acid. 
Table $3 \mid$ MS data of glycopeptides of human serum lgG detected in this study

\begin{tabular}{|c|c|c|c|c|c|c|c|}
\hline \multirow[b]{2}{*}{ Glycan $^{a}$} & \multirow[b]{2}{*}{ Structure } & \multicolumn{2}{|c|}{$\operatorname{lgG1}$ P01857 b EEQYN 297 STYR $^{b}$} & \multicolumn{2}{|c|}{$\operatorname{lgG} 2 / \operatorname{lgG} 3 \mathrm{P01859}{ }^{b} \mathrm{EEQFN}_{297}$ STFR $^{b}$} & \multicolumn{2}{|c|}{$\operatorname{lgG} 4$ P01861 bEEQFN 297 STYR $^{b}$} \\
\hline & & M & {$[\mathrm{M}+3 \mathrm{H}]^{3+}$} & M & {$[\mathrm{M}+3 \mathrm{H}]^{3+}$} & M & {$[\mathrm{M}+3 \mathrm{H}]^{3+}$} \\
\hline $\begin{array}{l}\text { Peptide only } \\
\text { GO } \\
\text { GON }\end{array}$ & :-... & $\begin{array}{l}1188.5047 \\
2486.9807 \\
2690.0615\end{array}$ & $\begin{array}{l}397.1682 \\
830.0008 \\
897.6940\end{array}$ & $\begin{array}{l}1156.5149 \\
2454.9909 \\
2658.0717\end{array}$ & $\begin{array}{l}386.5050 \\
819.3376 \\
887.0307\end{array}$ & $\begin{array}{l}1172.5098 \\
2470.9858 \\
2674.0666\end{array}$ & $\begin{array}{l}391.8366 \\
824.6692 \\
892.3623\end{array}$ \\
\hline GOFN & :-: & 2836.1180 & 946.3799 & 2804.1282 & 935.7167 & 2820.1231 & 941.0483 \\
\hline $\begin{array}{l}\text { GOF } \\
\text { GI } \\
\text { GIN }\end{array}$ & (50.: & $\begin{array}{l}2633.0386 \\
2649.0335 \\
2852.1141\end{array}$ & $\begin{array}{l}878.6868 \\
884.0184 \\
951.7116\end{array}$ & $\begin{array}{l}2601.0488 \\
2617.0437 \\
2820.1243\end{array}$ & $\begin{array}{l}868.0235 \\
873.3552 \\
941.0483\end{array}$ & $\begin{array}{l}2617.0437 \\
2633.0386 \\
2836.1192\end{array}$ & $\begin{array}{l}873.3552 \\
878.6868 \\
946.3799\end{array}$ \\
\hline GIFN & & 2998.1708 & 1000.3975 & 2966.1810 & 989.7343 & 2982.1759 & 995.0659 \\
\hline GIFNS &.+ & 3289.2662 & 1097.4293 & 3257.2764 & 1086.7661 & 3273.2713 & 1092.0977 \\
\hline GINS & $\because$ & 3143.2083 & 1048.7434 & 3111.2197 & 1038.0801 & - & - \\
\hline GIF & & 2795.0914 & 932.7044 & 2763.1016 & 922.0411 & 2779.0965 & 927.3728 \\
\hline GIFS & . & 3086.1869 & 1029.7362 & 3052.1970 & 1019.0729 & 3070.1919 & 1024.4046 \\
\hline G2 & 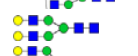 & 2811.0864 & 938.0361 & 2779.0965 & 927.3728 & 2795.0914 & 932.7044 \\
\hline $\mathrm{G} 2 \mathrm{~N}$ & & 3014.1669 & 1005.7292 & 2982.1771 & 995.0659 & 2998.1720 & 1000.3975 \\
\hline G2FN & & 3160.2236 & 1054.4151 & 3128.2338 & 1043.7519 & 3144.2287 & 1049.0835 \\
\hline G2NS & & 3305.2624 & 1102.7610 & - & - & - & - \\
\hline G2F & : & 2957.1443 & 986.7220 & 2925.2544 & 976.0587 & 2941.1494 & 981.3904 \\
\hline G2FS & - :-... & 3248.2397 & 1083.7538 & 3216.2499 & 1073.0906 & 3232.2448 & 1078.4222 \\
\hline $\begin{array}{l}\text { G2S } \\
\text { G2S2 }\end{array}$ & :10." & 3102.1818 & 1035.0679 & $\begin{array}{l}3070.1919 \\
3361.2874\end{array}$ & $\begin{array}{l}1024.4046 \\
1121.4364\end{array}$ & 3086.1869 & 1029.7362 \\
\hline Man8 & :-8.:. & 2891.0861 & 964.7026 & 2859.0963 & 954.0394 & 2875.0912 & 959.3710 \\
\hline Man9 & $\because 30$ & 3053.1389 & 1018.7202 & 3021.1491 & 1008.0570 & 3037.1440 & 1013.3886 \\
\hline Man9Glcl & $\because 8$ & 3215.1917 & 1072.778 & - & - & - & - \\
\hline 0100 & :.: & - & - & 2194.8902 & 732.6373 & 2210.8851 & 737.9689 \\
\hline 1000 & - & 2283.9015 & 762.3077 & 2251.9117 & 751.6444 & 2267.9066 & 756.9761 \\
\hline 1010 & 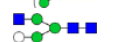 & 2445.9543 & 816.3253 & 2413.9645 & 805.6620 & 2429.9592 & 810.9937 \\
\hline 1100 & & 2429.9594 & 810.9937 & 2397.9696 & 800.3304 & 2413.9645 & 805.6620 \\
\hline 1110 & : & 2592.0122 & 865.0113 & 2560.0224 & 854.3480 & 2576.0173 & 859.6797 \\
\hline 1111 & : & & & 2851.1178 & 951.3798 & & - \\
\hline
\end{tabular}

tures'. Some genes [e.g., those encoding the calcium/calmodulindependent protein kinase type IV (CaMKIV), complement Clq subcomponent subunit $\mathrm{B}(\mathrm{Clqb})$, eukaryotic translation initiation factor 2 alpha kinase 4, and B-cell growth factor TNFRS17] were identified as potential biomarkers predicting T-cell and B-cell responses to vaccination because they were significantly differentially expressed in the early stage of the induced immune response $e^{26,28}$. Although these biological signatures identified in the studies of early response significantly contributed to the understanding of the specific responses triggered by vaccination, their ability to predict the outcome of vaccination on the basis of baseline immunological markers has remained elusive ${ }^{8}$. Very recently, Tsang and colleagues used a systems biology approach to identify baseline immunological predictors of vaccine response. They described, for the first time, a set of baseline pre-vaccination parameters that were revealed to be predictive for the antibody response after vaccination ${ }^{29}$. These studies not only raised the prospect of monitoring immune status before vaccination $^{29}$, but also highlighted the need for higher levels of resolution and broader spectrum immune parameters.

Antibodies are major components of the immune system. Immunoglobulin $\mathrm{G}$ is the main antibody isotype existed in blood and extracellular fluid and protects the body from infection by binding many kinds of pathogens, such as representing viruses, bacteria and fungi. Recently, high baseline antibody titer was found to inversely correlate with the post-vaccination response, demonstrating that pre-existing antibodies to HA peptides are predictive to the vaccination response ${ }^{8,29}$. Glycosylation, which plays crucial role in the effector function of IgG, has been proven to be distinctively regulated for antigen-specific antibodies. Meanwhile, it was revealed that prevaccination $B$ and $T$ cell subpopulations were potential predictors of endpoint antibody response ${ }^{8,29}$. Given that distinct IgG glycosylation profiles in individuals might result from the different levels of expression and/or activities of glycan-processing enzymes ${ }^{16}$ or the selective expansion of particular subsets of plasma cells with aberrant levels or activities of the corresponding enzymes ${ }^{16,30,31}$, we assumed that difference in the glycosylation of IgG between responders and nonresponders might pre-existed before vaccination and thereby can serve as prediction signatures for the consequences of vaccination.

To achieve this, we used our well-established UHPLC-QqQ-MS (MRM mode) method, which analyzed glycans at the glycopeptide level and thereby allowed the analysis of subclass-specific glycosylation, for the glycosylation analyses. The use of the MRM mode facilitates high sensitive quantification with a wide linear range. In total, 74 glycopeptides from four subclasses of IgG were quantitatively examined, confirming that our method is more comprehensive than the method used in a previous vaccination study, which detected about 50 glycoforms $\mathrm{s}^{25}$. Thus, the glycosylation of more glycoforms can be monitored with this high-performance method. On the other hand, unlike a previous study that investigated antigen-specific $\mathrm{IgGs}^{25}$, our study focused on the analysis of global glycans of the total IgGs in circulation. Antigen-specific IgGs have been shown to be glycosylated in very distinct ways, which differs considerably from the glycosylation of the pool of total IgGs ${ }^{32,33}$. By contrast, glycosylation of total IgGs is a combination of genetic background and all 
PCA
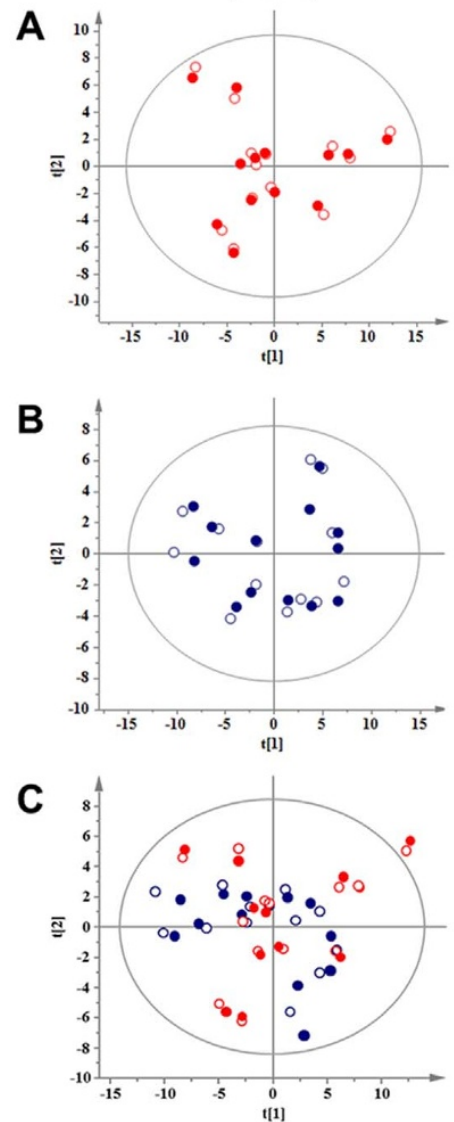

Nonresponder

- Before vaccination

- After vaccination
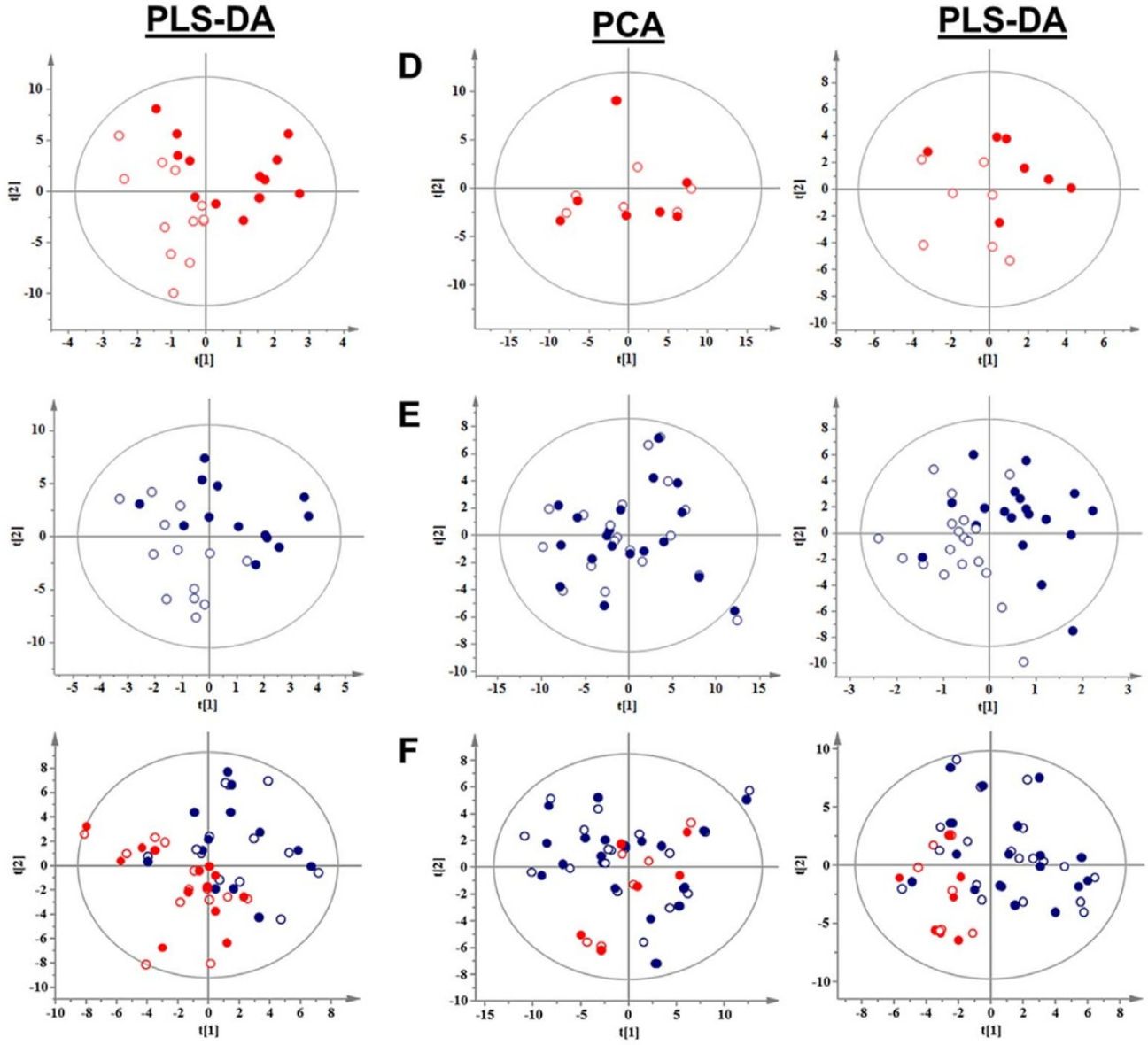

\section{Responder}

\section{- Before vaccination (Applies to all panels)}

Figure 3 PCA and PLS-DA scores plot of the glycosylation profiles of subjects before and after vaccination. (A-C) Analyses based on the antibody response to B strain. (D-F) Analyses based on antibody response to H1 strain.

relevant past events in the cell, hence can reflect the immune status of individual subjects more effectively.

In the previous study by Selman et al., the $\mathrm{N}$-glycosylation of antigen-specific IgG showed increased galactosylation and sialylation after influenza or tetanus vaccination, whereas the total IgGs showed no significant changes after vaccination ${ }^{25}$. Consistent with that study, our results showed no significant glycosylation changes in total IgGs after vaccination. Furthermore, the composition of the glycoforms of any individual subjects was found to be very stable over a time span of 14 days of pre- and post-vaccination, despite the high inter-subject variability. This phenomena indicated genetic, including epigenetic, regulation of IgG glycosylation, as evidenced in previous study of Gordan Lauc ${ }^{34}$. Because of this, differences in the glycosylation profiles of responders and nonresponders were observed in both pre- and post-vaccination samples, which suggested the potential of glycosylation profile as predictive signatures for the vaccination response. In addition, although the baseline $\mathrm{HI}$ titers were reversely correlated with post-vaccination response, the differentially expressed $\mathrm{Fc}$ glycosylation pattern between responders and nonresponders was not directly correlated to baseline antibody titer. Thus, the correlation between vaccination response and Fc glycosylation may not be simply driven by cross-correlation with pre-vaccination titers, indicating that the glycoform markers could potentially predict post-vaccination response independent of baseline antibody titers.
Characterization of differentially expressed glycoforms between responders and nonresponders to FluB revealed glycoform-specific changes of all three subclasses of IgGs (Figure 6). For example, three truncated glycoforms, i.e., 1010, 1110 and 1100 were found to be differentially expressed in responders and nonresponders to FluB (Figure 6), but their changes were not in the same trend. The relative abundance of glycoform 1010 in responders was about 4 folds lower than that in nonresponders, whereas the level of 1110 and 1100 were both higher in responders than in nonresponders. This kind of glycoform-specific changes implicated a kind of strictly and subtly regulated glycosylation of IgG. Further analysis of the potential glycoform markers revealed two interesting phenomena. One is the lower levels of IgG1-G0FN (a kind of bisected glycan) and concomitant higher levels of IgG1-G1FN and G2FN in responders (Figure 6), directing a relatively higher galactosylation level of the bisected glycans in responders. Another phenomenon is the overall higher levels of high mannose glycoforms in responders, including Man8, Man9 and Man9Glc1 of IgG1, as well as Man8 and Man9 of IgG2. Galactosylation is one of the most studied glycosylation feature of glycoprotein. Incomplete galactosylation of IgG could activate complement through the mannose-binding protein and therefore be involved in several pathological mechanisms ${ }^{34}$. Whereas high mannose glycoforms have been shown to have a significant impact on clinical efficacy and pharmacokinetics of therapeutic antibodies ${ }^{35}$. However, implications of the differentially expressed levels of these 

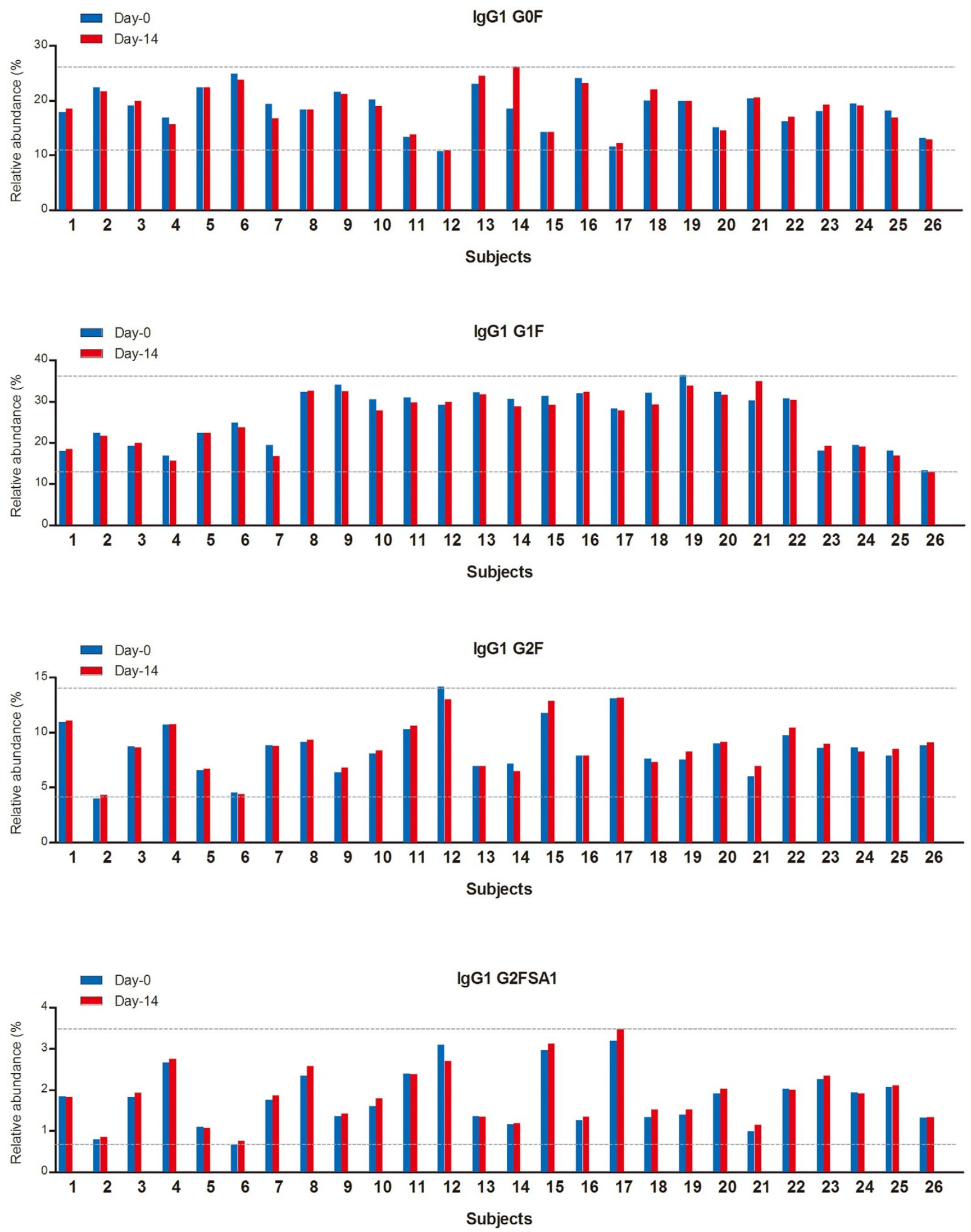

Figure $4 \mid$ Inter-subject variation of the relative abundance of representative glycoforms. 

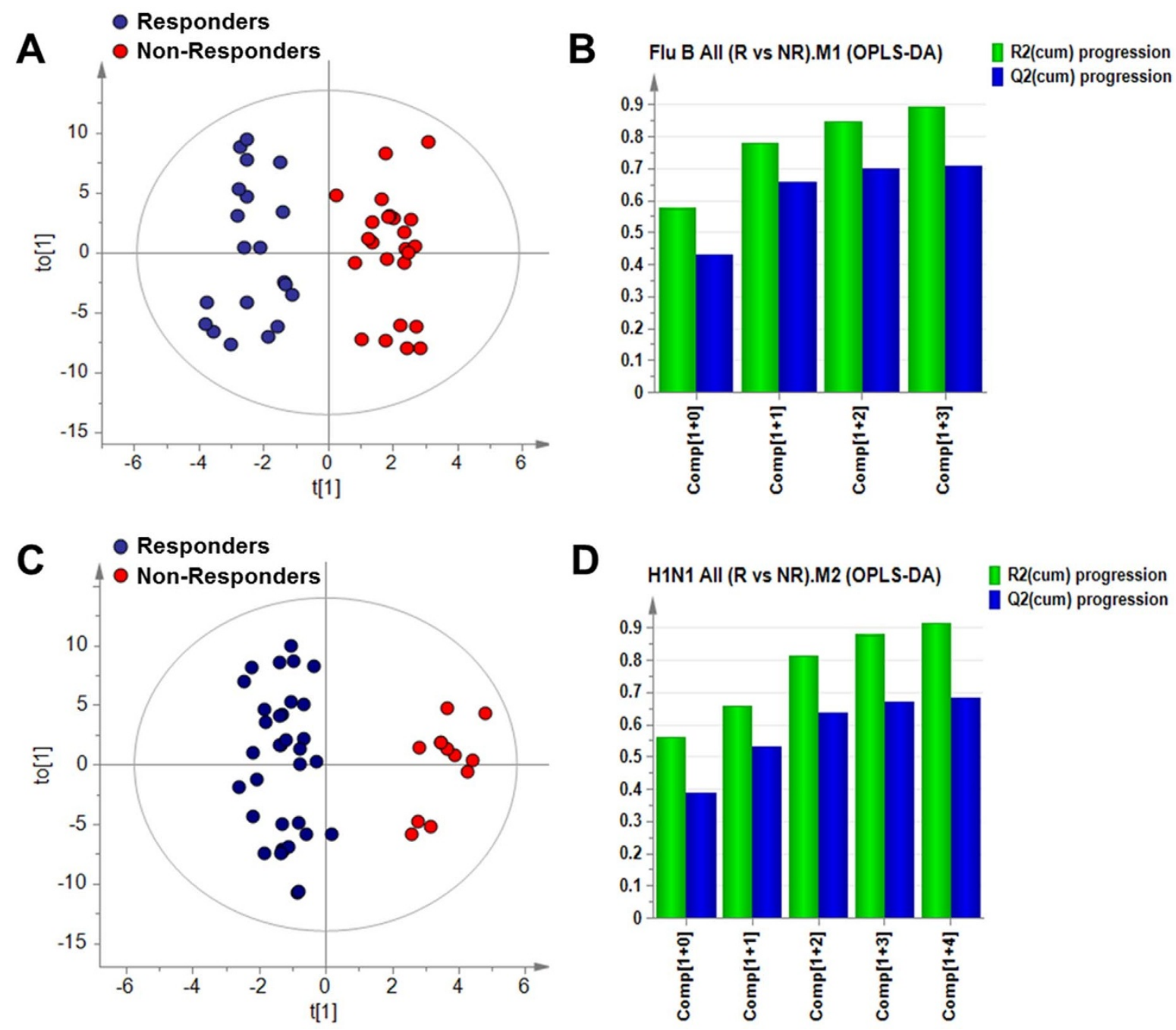

Figure $5 \mid$ Multivariate statistical results for IgG glycosylation in responders and nonresponders to FluB (A,B) and H1N1 (C,D) (HI test). (A,C) OPLSDA scores plot; $(\mathrm{B}, \mathrm{D})$ cumulative $\mathrm{Q}^{2}$ plot.

glycoforms between responders and nonresponders need to be explored in the future.

In addition to above features, a notable difference in the level of IgG2-G2 between responders and nonresponders was observed. This glycoform is featured by the lack of core-fucose which is attached to the first $\mathrm{N}$-acetylglucosamine in the core of the $\mathrm{N}$-glycan. Corefucose could modify the conformation of $\mathrm{Fc}$ region and lead to dramatically decreased binding ability of Fc to Fc $\gamma$ RIIIa $^{11,36}$, an activating Fc receptor expressed on natural killer (NK) cells. Binding of IgG to this receptor would result the initiation of ADCC. In this way, core-fucose functions as a 'safe switch' to prevent the elicitation of $\mathrm{ADCC}^{36}$. Glycoforms without core fucose are thus over 100 times more effective in triggering ADCC than fucosylated glycoforms ${ }^{34}$. Since ADCC has been proved to be an important pathway for the host to clear virus-infected cells, the higher level in IgG2-G2 in responders might indicate higher protective ability of these individuals against virus infection ${ }^{37}$. Another unusual glycosylation marker is IgG1-G1S1, which is a sialylated glycan. Terminal sialic acid on $\mathrm{N}$-glycan practically reverse function of IgG and change it from proinflammatory into anti-inflammatory agent. It has been elucidated that specific intercellular adhesion molecule-3-grabbing nonintegrin-R1 (SIGN-R1), a C-type lectin receptor is the target of sialylated IgG and mediates its anti-inflammatory effects ${ }^{38,39}$. Thus the lower level of G1S1 in nonresponders might reflect the lower anti-inflammatory ability of these subjects.
Similar to those observed for response to FluB, the glycoform signatures for the prediction of response to $\mathrm{H} 1 \mathrm{~N} 1$ were majorly involved bisected glycoforms and high mannose glycoforms (Figure 7). However, truncated glycoforms didn't show significant difference between responders and nonresponders to H1N1. This implied antigen-specific glycosylation signatures for TIV vaccination.

It should be noted that most of the predictive glycoform markers were of low-abundance species, of which the relative levels were generally below $10 \%$ or even $1 \%$. Without monitoring these lowabundance glycoforms, the predictive model of glycosylation on vaccination efficiency may not be established. Thus, 'comprehensive' glycomic approach with capability of analyzing low-abundance glycoforms was crucial for revealing the predictive capacity of Fc glycosylation for vaccination response.

It has been demonstrated that the glycosylation profile of IgG is tightly regulated by physiological and pathological determinants in an antigen-specific manner ${ }^{33,40}$. Different from antigen-specific antibodies, the composition of an individual's glycome is a combination of genetic background and all relevant past events in the cell ${ }^{34}$. The distinct total IgG glycosylation profiles in individuals observed in our study might result from the different levels of expression and/or activities of glycan-processing enzymes ${ }^{16}$ or the selective expansion of particular subsets of plasma cells with aberrant levels or activities of the corresponding enzymes ${ }^{30}$. Another possibility is that these individuals have encountered cross-reactive influenza strains during 

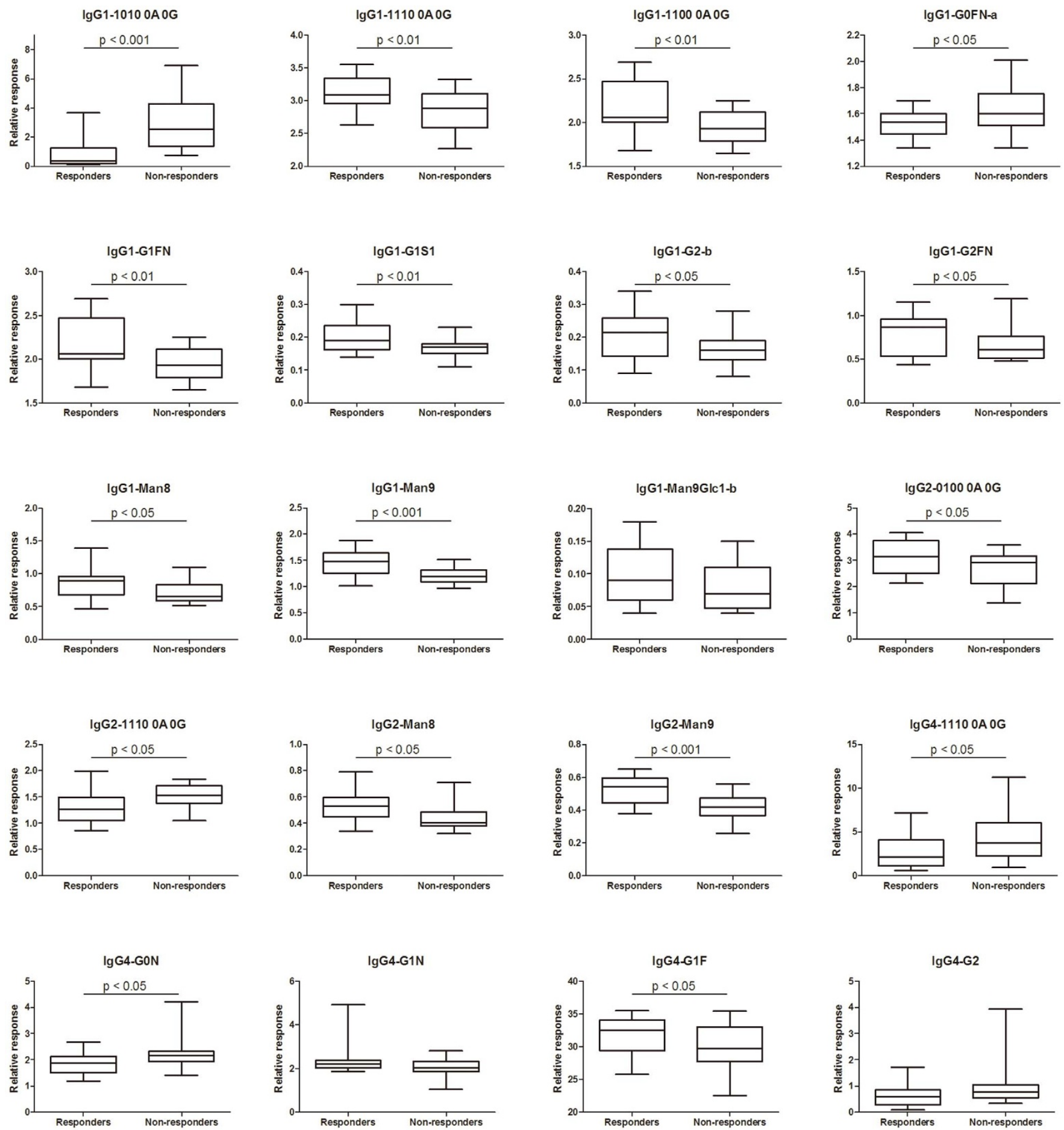

Figure 6 Box plots depicting changes in the relative abundances of the potential glycoforms signatures for responders $(n=13)$ and nonresponders $(n=13)$ to FluB.

previous infections or before the current vaccination. The different glycosylation patterns observed between responders and nonresponders might be the result of a combination of these factors. The mechanism underlying the predictive capacity of glycosylation profiles for antibody titers requires further exploration. It should be noted that the baseline state of antibody glycosylation profile among individuals is likely to reflect age, at least to some extent. Thus, the strength with which these predictors apply to individuals in different age categories remains to be determined.

In summary, this is the first study of serum IgG glycosylation profiling in healthy adults receiving a TIV. A multivariate analysis of this glycosylation revealed distinct glycosylation profiles in responders and nonresponders to both FluB and H1N1. Glycosylation markers that distinguishing responders and nonresponders were identified and validated. These IgG glycan markers can be used as molecular signatures to predict antibody titers after vaccination. The relative abundance of glycoforms contributing to prediction were stable within subjects over a time span of 14 days and had up to 4 folds difference between responders and nonresponders, and thus were likely predictive because they delineated distinct baseline immune states among individuals ${ }^{29}$. Our findings raise the possibility of using such approach to discover as biomarkers of immune response potential in the clinic. 

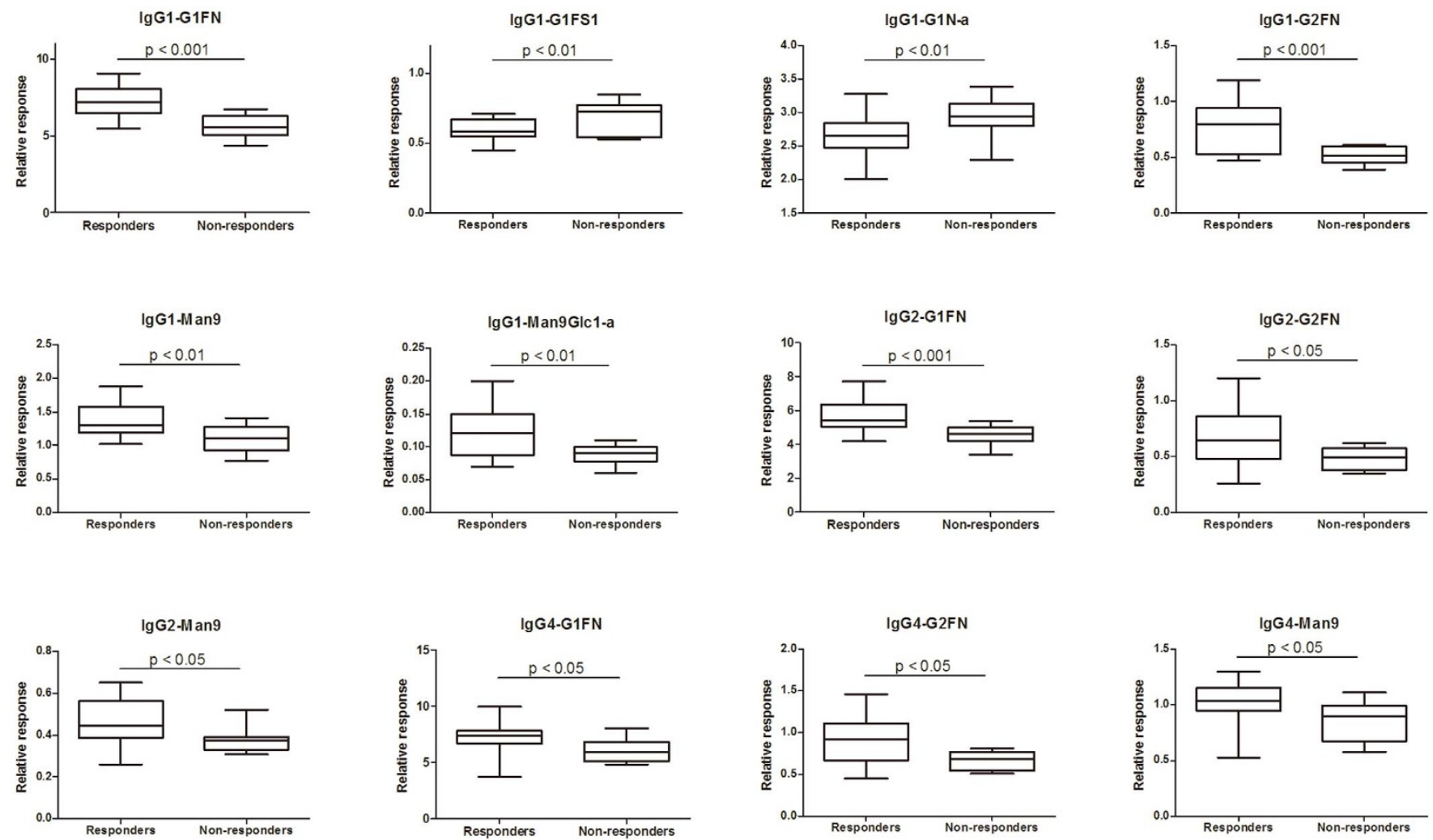

Figure 7 Box plots depicting changes in the relative abundances of the potential glycoform signatures for responders $(n=19)$ and non-responders $(n=7)$ to H1N1.

\section{Methods}

This study was approved by the Ethics Committee of the First Affiliated Hospital of Guangzhou Medical University, and all subjects signed an informed consent form. The methods were carried out in accordance with the approved guidelines.

Sample collection. Twenty-six healthcare workers from the First Affiliated Hospital of Guangzhou Medical University were administered the TIV in March 2011. All the subjects were healthy and were not taking any regular medication. Serum samples were collected from the participants before and 14 days after vaccination.

The TIV contained the following strains, which were recommended by WHO as the influenza vaccines for use in the 2010-2011 and 2011-2012 northern-hemisphere influenza seasons: A/California/7/2009 (H1N1)-like virus, A/Perth/16/2009 (H3N2)like virus, and B/Brisbane/60/2008-like virus.

Hemagglutination inhibition test (HI). The strains A/California/07/2009 (H1N1)like virus, A/Perth/16/2009 (H3N2)-like virus, and B/Brisbane/60/2008-like virus, to be used as antigens, were isolated from clinical samples by our laboratory and identified by sequencing their hemagglutinin and neuraminidase genes. All serum samples were tested by HI, as previously described ${ }^{41}$. Briefly, serum samples and red blood cells from chickens (Genebase Co., Guangzhou, China) were treated with receptor-destroying enzyme (Denka Seiken Co., Tokyo, Japan), according to the instructions for eliminating non-specific agglutinins and inhibitors before testing. All samples were tested in twofold dilutions from $1: 10$ to $1: 1280$. The highest dilution of serum that prevents hemagglutination is considered to be the HI titer of the serum.

Questionnaire. A survey was conducted by questionnaire in June of 2012. All the vaccine recipients enrolled in the study were asked whether they had developed influenza-like symptoms or had been hospitalized in the year after their inoculation. The questionnaire was administered to the enrolled subjects, all of whom answered all the questions, and all the information was treated confidentially.

IgG purification from serum with Protein G. Total IgGs were isolated from human serum with Protein G Sepharose ${ }^{\mathrm{TM}} 4$ Fast Flow beads $(90 \mu \mathrm{m}$; GE Healthcare, Uppsala, Sweden). The Protein G beads $(50 \mu \mathrm{L})$ were firstly washed twice with $250 \mu \mathrm{L}$ of binding buffer ( $20 \mathrm{mM}$ sodium phosphate, $\mathrm{pH}$ 7.0). The mixture including the beads, $250 \mu \mathrm{L}$ of binding buffer, and $10 \mu \mathrm{L}$ of serum was then mixed by inversion and incubated at room temperature for $1 \mathrm{~h}$. After incubation, the beads were washed twice with $250 \mu \mathrm{L}$ of binding buffer. The captured IgGs were eluted twice with $200 \mu \mathrm{L}$ of elution buffer (0.1 M glycine buffer, $\mathrm{pH} 2.7)$ and then neutralized with $30 \mu \mathrm{L}$ of neutralizing buffer (1 M Tris-HCl, $\mathrm{pH}$ 9.0). The buffer from the captured IgGs was exchanged with trypsin digestion buffer $(50 \mathrm{mM}$ ammonium bicarbonate in $15 \%$ acetonitrile, $\mathrm{pH} 7.4$ ) and concentrated to a final volume of $30 \mu \mathrm{L}$ with a $30 \mathrm{~K}$ centrifugal filter unit (Millipore, Carrigtwchill, County Cork, Ireland).

IgG digestion with trypsin. Trypsin Gold (mass spectrometry grade; Promega, Wisconsin, USA) was first reconstituted in $50 \mathrm{mM}$ acetic acid to a final concentration of $1 \mu \mathrm{g} / \mu \mathrm{L}$ and then further diluted to $0.05 \mu \mathrm{g} / \mu \mathrm{L}$ with trypsin digestion buffer. The trypsin solution $(10 \mu \mathrm{L})$ was added to the IgG samples and the mixtures were diluted to a final volume of $100 \mu \mathrm{L}$ with trypsin digestion buffer and incubated overnight at $37^{\circ} \mathrm{C}$. After incubation, $0.5 \mu \mathrm{L}$ of formic acid was added to quench the digestion reaction. The samples were centrifuged at $14000 \mathrm{~g}$ for $5 \mathrm{~min}$, and the supernatant was stored at $-80^{\circ} \mathrm{C}$ until analysis.

Ultrahigh-performance liquid chromatography (UHPLC)-mass spectrometry (MS) analysis of glycopeptides. UHPLC was performed with an Agilent 1290 Infinity UHPLC system (Agilent Technologies, Santa Clara, CA, USA), equipped with a binary solvent delivery system and a standard autosampler. Chromatography was performed on an Agilent ZORBAX Eclipse Plus $\mathrm{C}_{18}$ column $(2.1 \times 150 \mathrm{~mm}$, $1.8 \mu \mathrm{m}$ ), and the column temperature was maintained at $40^{\circ} \mathrm{C}$. The mobile phase consisted of $0.1 \%$ formic acid in distilled water $(\mathrm{A})$ and $0.1 \%$ formic acid in acetonitrile (B). The following optimized linear gradient was applied: 0-8 min, $2 \%-$ 6\% B; 8-16 min, 6\%-10\% B; 16-20 min, $10 \%-35 \%$ B; $20-25$ min, $35 \%-50 \%$ B; $25-$ $30 \mathrm{~min}, 50 \%-100 \% \mathrm{~B} ; 30-32 \mathrm{~min}, 100 \% \mathrm{~B}$; followed by the equilibration of the column for $8 \mathrm{~min}$ before the next run. The flow rate was $0.3 \mathrm{~mL} / \mathrm{min}$ and the injection volume was $5 \mu \mathrm{L}$.

The UHPLC system was coupled to an Agilent 6490 Triple Quadrupole LC/MS System (Agilent Technologies) equipped with an iFunnel and an Electrospray Ion Source (ESI) with Agilent Jet Stream Technology. Nitrogen was used as the nebulizing gas ( $40 \mathrm{psi})$, the drying gas $\left(16 \mathrm{~L} / \mathrm{min}, 250^{\circ} \mathrm{C}\right.$ ), and the sheath gas $\left(11 \mathrm{~L} / \mathrm{min}, 400^{\circ} \mathrm{C}\right)$. The capillary voltage was set to $4000 \mathrm{~V}$ and the nozzle voltage to $1500 \mathrm{~V}$. The mass analyzer was operated in positive mode and in the multiple reaction monitoring (MRM) mode with a dwell time of $10 \mathrm{~s}$, a fragmentor voltage of $380 \mathrm{~V}$, and a collision energy of $18 \mathrm{~V}$. The triplely charged ions $[\mathrm{M}+3 \mathrm{H}]^{3+}$ of the glycopeptides (Table 3) were set as precursor ions, and fragment ions generated from $\mathrm{N}$-acetylglucosamine (GlcNAc) at $m / z 204.1$ or hexose-GlcNAc at $m / z 366.1$ were employed as product ions for MRM transitions.

Multivariate analysis. All samples were prepared in duplicate and subjected to LCMS analyses in duplicate. The samples were analyzed using the software of Agilent MassHunter Quantitative Analysis (version B.05.00, Agilent Technologies). The intensity of each peak obtained was normalized as the percentage peak intensity relative to the total peak intensity in that sample. Multivariate statistical data analyses, including a principal components analysis (PCA), partial least-squares-discriminant 
analysis (PLS-DA), and orthogonal partial least squares discriminate analysis (OPLSDA), were performed using the SIMCA 13.0 software (Umetrics, Umea, Sweden). The scores plot of the PCA displays the general clustering, trends, or outliers in the observations (or samples), whereas PLS-DA and OPLS-DA are supervised methods capable of removing information from an input data set $\mathrm{X}$ that is unrelated to the response set $\mathrm{Y}$ to improve the interpretation of the variations responsible for the separation. The $\mathrm{R}^{2} \mathrm{Y}$ and $\mathrm{Q}^{2} \mathrm{Y}$ values indicate the goodness of the OPLS-DA model. $R^{2} Y$ provides an estimate of how well the model fits the $Y$ data, whereas $Q^{2} Y$ is an estimate of how well the model predicts $\mathrm{Y}$. Cumulative values of $\mathrm{R}^{2} \mathrm{Y}$ and $\mathrm{Q}^{2} \mathrm{Y}$ close to 1 indicate an excellent model.

The major differences in glycosylation between the responders and nonresponders were determined based on loading plots and the variable importance parameters (VIPs) for each pattern recognition model. The loading plots display the correlation between the $\mathrm{X}$ variables, in the first dimension, or the residuals of the $\mathrm{X}$ variables in subsequent dimensions. The VIP indicates the effect on class membership. A VIP value larger than 1 is more successfully explains $Y$ than smaller VIP values. In general, the glycosylation that contributed to the variation in the present study was identified by ranking the VIP values, and further validated with a univariate analysis (Student's t test).

Statistical analysis. The statistical software SPSS 13.0 (SPSS, Chicago, IL, USA) was used for all statistical analyses. Quantitative characteristics are presented as means \pm SD. Data that were normally distributed were compared using analysis of variance (ANOVA). All hypothesis testing was two-sided and $\mathrm{P}<0.05$ was defined as significant.

1. World-Health-Organization. Vaccine use. http://www.who.int/influenza/ vaccines/use/en/ (2014) 15/05/2014.

2. Osterholm, M. T., Kelley, N. S., Sommer, A. \& Belongia, E. A. Efficacy and effectiveness of influenza vaccines: a systematic review and meta-analysis. Lancet Infect. Dis. 12, 36-44 (2012).

3. Sasaki, S. et al. Influence of prior influenza vaccination on antibody and B-cell responses. PLoS One 3, e2975 (2008).

4. Oxford, J. S., Al-Jabri, A. A., Lambkin, R., Palache, A. M. \& Fleming, D. M. Nonresponders to egg grown influenza vaccine seroconvert after booster immunization with MDCK cell grown vaccine. Vaccine 21, 2743-2746 (2003).

5. Eiselt, J., Kielberger, L., Sedlackova, T., Racek, J. \& Pazdiora, P. High ferritin, but not hepcidin, is associated with a poor immune response to an influenza vaccine in hemodialysis patients. Nephron Clin. Pract. 115, c147-153 (2010).

6. Lambkin, R., Novelli, P., Oxford, J. \& Gelder, C. Human genetics and responses to influenza vaccination: clinical implications. Am. J. Pharmacogenomics 4, 293-298 (2004).

7. Gelder, C. M. et al. Associations between human leukocyte antigens and nonresponsiveness to influenza vaccine. J. Infect. Dis. 185, 114-117 (2002).

8. Miller, M. S. \& Palese, P. Peering into the crystal ball: influenza pandemics and vaccine efficacy. Cell 157, 294-299 (2014).

9. Ravetch, J. V. \& Bolland, S. IgG Fc receptors. Annu. Rev. Immunol. 19, 275-290 (2001).

10. Lutz, H. U. How immune complexes from certain IgG NAbs and any $\mathrm{F}\left(\mathrm{ab}^{\prime}\right)(2) \mathrm{can}$ mediate excessive complement activation. Adv. Exp. Med. Biol. 750, 186-196 (2012).

11. Shields, R. L. et al. Lack of fucose on human IgG1 N-linked oligosaccharide improves binding to human Fcgamma RIII and antibody-dependent cellular toxicity. J. Biol. Chem. 277, 26733-26740 (2002).

12. Malhotra, R. et al. Glycosylation changes of IgG associated with rheumatoid arthritis can activate complement via the mannose-binding protein. Nat. Med. 1, 237-243 (1995).

13. Kaneko, Y., Nimmerjahn, F. \& Ravetch, J. V. Anti-inflammatory activity of immunoglobulin G resulting from Fc sialylation. Science 313, 670-673 (2006).

14. Umana, P., Jean-Mairet, J., Moudry, R., Amstutz, H. \& Bailey, J. E. Engineered glycoforms of an antineuroblastoma IgG1 with optimized antibody-dependent cellular cytotoxic activity. Nat. Biotechnol. 17, 176-180 (1999).

15. Kobata, A. The N-linked sugar chains of human immunoglobulin G: their unique pattern, and their functional roles. Biochim. Biophys. Acta 1780, 472-478 (2008).

16. Wang, J. et al. Fc-glycosylation of IgG1 is modulated by B-cell stimuli. Mol. Cell. Proteomics 10, M110 004655 (2011).

17. Schnaar, R. et al. Program and abstracts for the 2012 Joint Meeting of the Society for Glycobiology \& American Society for Matrix Biology. Glycobiology 22, 1518-1650 (2012).

18. Parekh, R. B. et al. Association of rheumatoid arthritis and primary osteoarthritis with changes in the glycosylation pattern of total serum IgG. Nature 316, 452-457 (1985)

19. Kuroda, Y. et al. Structural studies on IgG oligosaccharides of patients with primary Sjogren's syndrome. Glycoconj. J. 19, 23-31 (2002).

20. Wuhrer, M. et al. Glycosylation profiling of immunoglobulin $\mathrm{G}$ (IgG) subclasses from human serum. Proteomics 7, 4070-4081 (2007).

21. van de Geijn, F. E. et al. Immunoglobulin G galactosylation and sialylation are associated with pregnancy-induced improvement of rheumatoid arthritis and the postpartum flare: results from a large prospective cohort study. Arthritis Res. Ther. 11, R193 (2009).

22. Martin, K., Talukder, R., Hay, F. C. \& Axford, J. S. Characterization of changes in IgG associated oligosaccharide profiles in rheumatoid arthritis, psoriatic arthritis, and ankylosing spondylitis using fluorophore linked carbohydrate electrophoresis. J. Rheumatol. 28, 1531-1536 (2001).
23. Bondt, A. et al. Changes in IgG-Fc N-glycan sialylation, galactosylation and fucosylation influence disease activity during and after pregnancy in rheumatoid arthritis. Ann. Rheum. Dis. 71, A34-A35 (2012).

24. Mesko, B. et al. Peripheral blood gene expression and IgG glycosylation profiles as markers of tocilizumab treatment in rheumatoid arthritis. J. Rheumatol. 39, 916-928 (2012)

25. Selman, M. H. et al. Changes in antigen-specific IgG1 Fc N-glycosylation upon influenza and tetanus vaccination. Mol. Cell. Proteomics : MCP 11, M111 014563 (2012).

26. Nakaya, H. I. et al. Systems biology of vaccination for seasonal influenza in humans. Nat. Immunol. 12, 786-795 (2011).

27. U.S. Department of Health and Human Services, Food and Drug Administration, Center for Biologics Evaluation and Research. Guidance for Industry Clinical: Data Needed to Support the Licensure of Seasonal Inactivated Influenza Vaccines. http:// www.fda.gov/BiologicsBloodVaccines/GuidanceComplianceRegulatoryInformation/ Guidances/Vaccines/ucm074794.htm (2007) 15/05/2014.

28. Querec, T. D. et al. Systems biology approach predicts immunogenicity of the yellow fever vaccine in humans. Nat. Immunol. 10, 116-125 (2009).

29. Tsang, J. S. et al. Global analyses of human immune variation reveal baseline predictors of postvaccination responses. Cell 157, 499-513 (2014).

30. Omtvedt, L. A. et al. Glycan analysis of monoclonal antibodies secreted in deposition disorders indicates that subsets of plasma cells differentially process IgG glycans. Arthritis Rheum. 54, 3433-3440 (2006).

31. Buchlis, G. et al. Enhanced T cell function in a mouse model of human glycosylation. J. Immunol. 191, 228-237 (2013).

32. Wuhrer, M. et al. Regulated glycosylation patterns of IgG during alloimmune responses against human platelet antigens. J.Proteome Res. 8, 450-456 (2009)

33. Scherer, H. U. et al. Glycan profiling of anti-citrullinated protein antibodies isolated from human serum and synovial fluid. Arthritis Rheum. 62, 1620-1629 (2010).

34. Gornik, O., Pavic, T. \& Lauc, G. Alternative glycosylation modulates function of IgG and other proteins - implications on evolution and disease. Biochim. Biophys. Acta 1820, 1318-1326 (2012)

35. Shi, H. H. \& Goudar, C. T. Recent advances in the understanding of biological implications and modulation methodologies of monoclonal antibody N-linked high mannose glycans. Biotechnol. Bioeng. 111, 1907-1919 (2014).

36. Kapur, R. et al. A prominent lack of IgG1-Fc fucosylation of platelet alloantibodies in pregnancy. Blood 123, 471-480 (2014).

37. Marasco, W. A. \& Sui, J. The growth and potential of human antiviral monoclonal antibody therapeutics. Nat. Biotechnol. 25, 1421-1434 (2007).

38. Schwab, I. \& Nimmerjahn, F. Intravenous immunoglobulin therapy: how does IgG modulate the immune system? Nat. Rev. Immunol. 13, 176-189 (2013).

39. Sondermann, P., Pincetic, A., Maamary, J., Lammens, K. \& Ravetch, J. V. General mechanism for modulating immunoglobulin effector function. Proc. Natl. Acad. Sci. USA 110, 9868-9872 (2013)

40. Saldova, R. et al. Ovarian cancer is associated with changes in glycosylation in both acute-phase proteins and IgG. Glycobiology 17, 1344-1356 (2007).

41. World-Health-Organization (eds). Serological diagnosis of influenza by haemagglutination inhibition testing. in Manual for the laboratory diagnosis and virological surveillance of influenza 59-62 (WHO Press, Switzerland, 2011).

\section{Acknowledgments}

This project is sponsored by Macao Science and Technology Development Fund (020/2013/ A1 to J.Z.H.) and financially supported by Emergency Response Project of Ministry of Science and Technology of China (Grant no.KJYJ-2013-01-05), National Science and Technology Major Project of the Ministry of Science and Technology of China (Grant no. 2014ZX10004006)

\section{Author contributions}

Z.H.J., J.R.W., Z.F.Y. and L.L. conceived and designed the experiments. W.D.G., L.F.Y., W.N.G. and Y.Q.Z. performed the experiments, and analyzed the data. J.R.W. and W.D.G. wrote the main manuscript text, W.D.G., L.F.Y. and J.R.W. prepared all figures. All authors reviewed the manuscript.

\section{Additional information}

Supplementary information accompanies this paper at http://www.nature.com/ scientificreports

Competing financial interests: The authors declare no competing financial interests. How to cite this article: Wang, J.-R. et al. Glycomic Signatures on Serum IgGs for Prediction of Postvaccination Response. Sci. Rep. 5, 7648; DOI:10.1038/srep07648 (2015).

This work is licensed under a Creative Commons Attribution-NonCommercialShareAlike 4.0 International License. The images or other third party material in this article are included in the article's Creative Commons license, unless indicated otherwise in the credit line; if the material is not included under the Creative Commons license, users will need to obtain permission from the license holder in order to reproduce the material. To view a copy of this license, visit http:// creativecommons.org/licenses/by-nc-sa/4.0/ 